\begin{tabular}{|c|l|}
\hline Title & Demographic Properties Shape Tree Size Distribution in a Mal aysian Rain Forest \\
\hline Author(s) & Kohyama, Takashi S.; Potts, Matthew D.; Kohyama, Tetsuo I.; Kassim, A bd Rahman; A shton, Peter S. \\
\hline Citation & $\begin{array}{l}\text { The A merican Naturalist, 185/3), 367-379 } \\
\text { https://doi.org/40.1086/679664 }\end{array}$ \\
\hline Issue Date & 2015-03 \\
\hline Doc URL & http://hdl.handle.net/2115/58220 \\
\hline Type & article \\
\hline File Information & Kohyama_etal_2015_A mNat.pdf \\
\hline
\end{tabular}

Instructions for use 


\title{
Demographic Properties Shape Tree Size Distribution in a Malaysian Rain Forest
}

\author{
Takashi S. Kohyama, ${ }^{1,2,}$ Matthew D. Potts, ${ }^{2,3}$ Tetsuo I. Kohyama, ${ }^{4}$ Abd Rahman Kassim, ${ }^{5}$ and \\ Peter S. Ashton ${ }^{2}$
}

1. Faculty of Environmental Earth Science, Hokkaido University, Sapporo 060-0810, Japan; 2. Harvard University Herbaria, Cambridge, Massachusetts 002138; 3. Department of Environmental Science, Policy, and Management, University of California, Berkeley, California 94720; 4. Faculty of Science, Hokkaido University, Sapporo 060-0810, Japan; 5. Forest Research Institute Malaysia, Kepong 52109, Malaysia Submitted April 24, 2014; Accepted November 6, 2014; Electronically published January 21, 2015

Dryad data: http://dx.doi.org/10.5061/dryad.bb460.

ABSTRACt: Different mechanisms have been proposed to explain how vertical and horizontal heterogeneity in light conditions enhances tree species coexistence in forest ecosystems. The foliage partitioning theory proposes that differentiation in vertical foliage distribution, caused by an interspecific variation in mortality-to-growth ratio, promotes stable coexistence. In contrast, successional niche theory posits that horizontal light heterogeneity, caused by gap dynamics, enhances species coexistence through an interspecific tradeoff between growth rate and survival. To distinguish between these theories of species coexistence, we analyzed tree inventory data for 370 species from the 50-ha plot in Pasoh Forest Reserve, Malaysia. We used community-wide Bayesian models to quantify size-dependent growth rate and mortality of every species. We compared the observed size distributions and the projected distributions from sizedependent demographic rates. We found that the observed size distributions were not simply correlated with the rate of population increase but were related to demographic properties such as size growth rate and mortality. Species with low relative abundance of juveniles in size distribution showed high growth rate and low mortality at small tree sizes and low per-capita recruitment rate. Overall, our findings were in accordance with those predicted by foliage partitioning theory.

Keywords: Bayesian model, coexistence, community ecology, demography, equilibrium, skewness, trade-off.

\section{Introduction}

Three-dimensional variation in light conditions caused by heterogeneity in stand structure has been proposed to enhance tree species coexistence in forest ecosystems (Kohyama 1993; Falster and Westoby 2005). According to the foliage partitioning theory (Kohyama and Takada 2009, 2012), one-sided competition for light driven by vertical

* Corresponding author; e-mail: kohyama@ees.hokudai.ac.jp.

Am. Nat. 2015. Vol. 185, pp. 367-379. (C) 2015 by The University of Chicago. 0003-0147/2015/18503-55443\$15.00. All rights reserved. DOI: $10.1086 / 679664$ foliage distribution coupled with interspecific variation in demographic properties (size growth, survival, and reproduction rates) promotes species coexistence. In contrast, the theory of successional niche (Pacala and Rees 1998; Rees et al. 2001), in relation to the trade-off between tree growth rate in full sunlight and survival in deep shade, posits that horizontal light heterogeneity caused by the disturbance and recovery of canopy structure enhances species coexistence. Determining the relative importance of these theories of species coexistence in diverse tree communities remains an open question.

The foliage partitioning theory posits two specific mechanisms of coexistence. First, species that differ in maximum adult stature can coexist stably when tall-statured species have high size growth rate but low fecundity (Kohyama and Takada 2009). Second, species with the same maximum adult stature but different tree size distributions can coexist stably (Kohyama and Takada 2012). Species with low mortality-to-growth and low fecundity exhibit stable size distributions with low skewness, whereas species with opposite demographic properties exhibit stable size distribution with high skewness (fig. 1A). King et al. $(2006 b)$ found that interspecific differentiation in maximum adult height characterizes species-rich tropical rain forests, suggesting the importance of the first mechanism. In addition, they showed that the majority of tree species in temperate forests have the same maximum adult height at the top of the canopy layer, suggesting the importance of the second mechanism. For example, in the subalpine forests of northeastern United States and northern Japan, top canopy spruce and fir species differ in their size distributions. Spruce has lower mortality and grows better in deep shade at the juvenile stage than fir, while fir has a higher recruitment rate (Shea 1985; Takahashi 1996; Takahashi and Kohyama 1999). It has yet to be investigated 

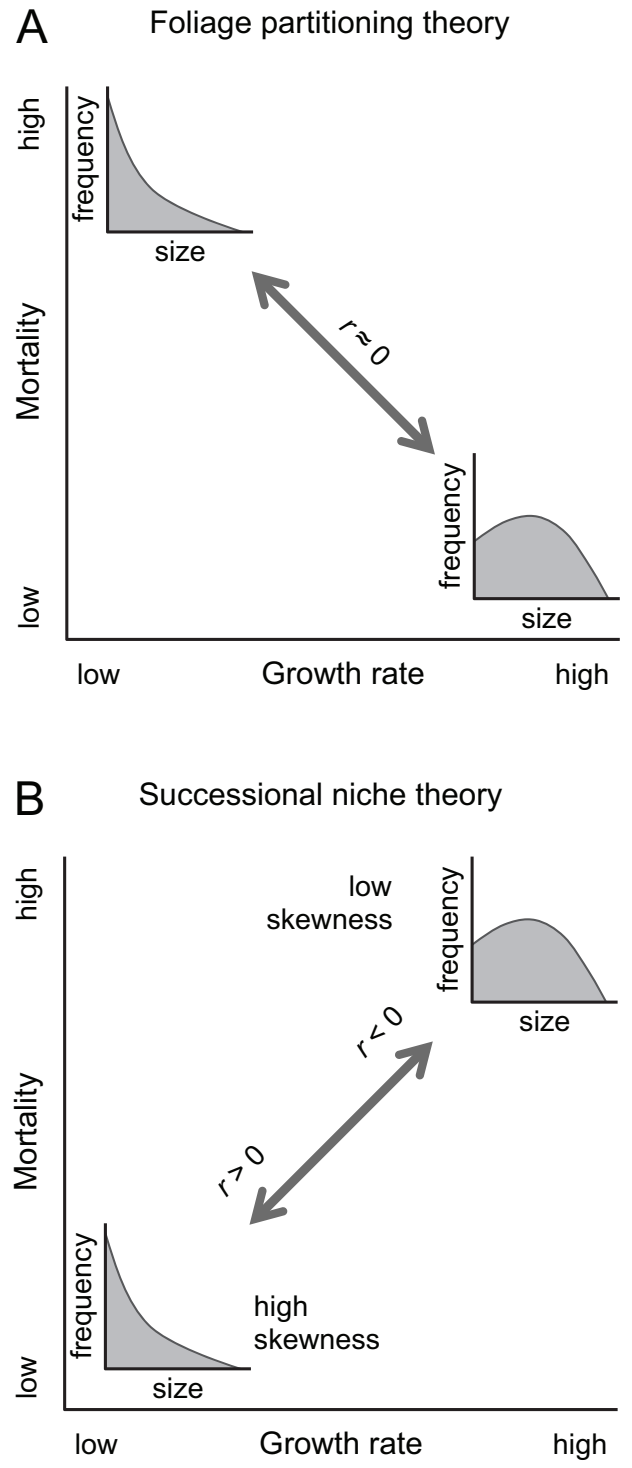

Figure 1: Alternative predictions for the linkage between the shape of the tree size distribution and demographic rates, illustrated on $\log$-log coordinates of mean size growth rate and mortality. Insets show expected size frequency distributions on the logarithmic size scale. $A$, The foliage partitioning theory of species coexistence posits interspecific differentiation in stationary size distribution in a stable forest tree community, in which species with a low mortality-togrowth ratio show less skewed size distributions. $B$, The successional niche theory posits the growth-survival trade-off, in which lightdemanding species with a high growth rate in gaps and low survival in deep shade fail to recruit and have low rates of population increase $(r)$ and less skewed size distributions in mature closed stands in horizontally heterogeneous forests.

whether the second mechanism (fig. 1A) occurs in speciesrich tropical tree communities.

In contrast to the interspecific differentiation in stable size distributions predicted by the foliage partitioning the- ory, successional niche theory (Pacala and Rees 1998) in relation to the growth-survival trade-off (also referred to as the growth-mortality trade-off) leads to a hypothesis of differentiation in transient size distributions between species. Wright et al. $(2003,2010)$ and Poorter et al. (2008) demonstrated for tropical forests the existence of interspecific trade-off between high growth rate in full sunlight for gap-demanding species and high survival probability in deep shade for shade-tolerant species. At a spatial scale of forest stands, light-demanding species that have established in past gaps fail to recruit in mature stands with dark forest floors and thus exhibit low relative abundance of juveniles in closed canopy stands. Conversely, shadetolerant species that are able to recruit on the dark forest floor exhibit size distributions with high juvenile abundance (fig. 1B). Even at a much larger spatial scale of a 50-ha plot in Barro Colorado Island (BCI) in Panama, which covers all phases of gap dynamics, Wright et al. (2003) showed that low skewness of species size distribution was associated with high size growth rate and high mortality at the juvenile stage. This implies that shadeintolerant species in the BCI forest experience population decline and are replaced by shade-tolerant species. However, Condit et al. (1998) showed that the shape of tree size distribution did not reflect the rate of population increase among abundant tree species in the BCI forest.

From the above, it is clear, especially for species-rich tropical rain forests, that more work is needed to evaluate the possible contributions of maximum size differentiation among species, differentiation in tree size distribution among species with the same maximum size, and the growth-survival trade-off in promoting species coexistence.

Distinguishing between these mechanisms (fig. 1) requires a detailed examination of the relationships between tree size distribution and demographic properties of tree species. While some previous studies (Kohyama 1992; Thomas 1996; Condit et al. 1998; Kohyama et al. 2003; Wright et al. 2003, 2010; King et al. 2006a, 2006b; Feeley et al. 2007; Poorter et al. 2008; Bin et al. 2012) have compared tree size distribution and demographic properties, they focused on either maximum size or the shape of size distribution but not both. In addition, a key limitation of these studies is that they estimated demographic parameters separately for each of some abundant species. The estimation of size dependence in growth and mortality is inevitably affected by the size distribution itself. It is therefore preferable for interspecific comparison to employ community-wide statistical procedures for estimating the demographic parameters of each species (Rüger et al. 2011a, 2011b; Iida et al. 2014).

In this study, we exhaustively examined the alternative expectations of interspecific linkages between demography and size distribution (displayed in fig. 1) for a species-rich 
tree community in the Pasoh 50-ha plot in Peninsular Malaysia. We quantified stem diameter distribution (the coefficient of skewness and upper 99th percentile diameter) and demographic rates (diameter growth rate, mortality, and recruitment rates) for all 370 species with $\geq 100$ trees (stem diameter of $\geq 2-\mathrm{cm}$ ) in the 50 -ha plot. Iida et al. (2011) previously showed that allometries between stem diameter and foliage-crown dimensions are similar across species in the Pasoh forest. Thus, we assume that interspecific differences in stem diameter distribution correspond to differentiation in vertical foliage distribution among species. To determine whether interspecific variations in stem diameter distribution and demographic properties merely represent sampling bias from a community-wide tree population, we developed a community with permuted species identity as a random assembly null model and compared the permuted community to the actual community. We employed community-wide hierarchical Bayesian statistics to estimate size-dependent growth and mortality parameters of every species. To determine whether species size distributions were related to species demographic properties or related to changes in species population size, we compared observed size distributions with projected size distributions based on estimated demographic rates. Finally, we determined to what extent demographic properties explained tree size distributions in relation to the specific predictions of the two alternative theories (fig. 1).

\section{Demographic Projection of Size Distribution}

To project tree size distribution from demographic fates, we employed a one-dimensional continuity equation from fluid dynamics (VanSickle 1977; Kohyama 1992, 1993). Change in stem diameter distribution with time of a tree species population per unit area $f(t, x)\left(\mathrm{cm}^{-1} \mathrm{ha}^{-1}\right)$ at stem diameter $x(\mathrm{~cm})$ at time $t$ (year) is described by the instantaneous tree mortality $M(t, x)$ (year $\left.{ }^{-1}\right)$ and diameter growth rate $G(t, x)\left(\mathrm{cm} \mathrm{year}^{-1}\right)$ as follows:

$$
\frac{\partial f(t, x)}{\partial t}=-\frac{\partial[G(t, x) f(t, x)]}{\partial x}-M(t, x) f(t, x) .
$$

Equation (1) is the first-order approximation of a more general model with higher-order differentiation terms with respect to $x$ (Suzuki 1966; Hara 1984; Kohyama and Hara 1989), which takes into account the growth rate distribution at a given $x$. For equation $(1), G(t, x)$ corresponds to the arithmetic mean of the growth rate distribution at $x$ (Kohyama and Takada 1998; Kohyama et al 2005). The boundary condition of equation (1) provides the recruitment rate $B(t)\left(\mathrm{ha}^{-1}\right.$ year $\left.^{-1}\right)$ at the minimum size $x_{0}(\mathrm{~cm})$ :

$$
B(t)=G\left(t, x_{0}\right) f\left(t, x_{0}\right) .
$$

If mean growth rate and mortality of equation (1) are assumed to be independent of time $t$, the change in size distribution satisfies $\partial f(t, x) / \partial t=r f(t, x)$, where $r\left(\right.$ year $\left.^{-1}\right)$ is the intrinsic rate of population increase. Therefore, the frequency distribution of tree size is unchanged with $t$, such that

$$
\frac{1}{f(t, x)} \frac{\partial f(t, x)}{\partial x}=-\frac{1}{G(x)} \frac{\mathrm{d} G(x)}{\mathrm{d} x}-\frac{M(x)}{G(x)}-\frac{r}{G(x)} .
$$

Integrating equation (3) with respect to $x$ and using the boundary condition (2), we obtain

$$
f(t, x)=\frac{B(t)}{G(x)} \exp \left[-\int_{x_{0}}^{x} \frac{M(\hat{x})+r}{G(\hat{x})} \mathrm{d} \hat{x}\right]
$$

(Kohyama et al. 2003). In this study, we used equations (3) and (4) to project tree size distributions with demographic rates and compared predicted distributions with observed ones.

\section{Methods \\ Actual and Permuted Communities}

We analyzed inventory data from the 50-ha Forest Dynamics Plot in the Pasoh Forest Reserve located in Negeri Sembilan, Malaysia. The forest is representative of intact mixed dipterocarp forest of lowland Peninsular Malaysia and contains about 820 tree species (Condit et al. 1999). A plot census has been conducted at about 5 -year intervals since 1985 to record stem diameter at $1.3 \mathrm{~m}$ (or at marked position for trees with buttresses) to the nearest $0.1 \mathrm{~cm}$ for all trees with a diameter of $\geq 1 \mathrm{~cm}$. Census protocols are given in Condit (1998), and the tree flora of the plot is described in Kochummen (1997). We used data from the 1990 and 2000 censuses. We excluded monocot species without secondary growth of stems (seven species of palms, three Dracaena spp., and two Pandanus spp.) and species with populations of $<100$ individuals with a diameter of $\geq 2 \mathrm{~cm}$ and an upper tree size (defined as the 99th percentile diameter) of $<5 \mathrm{~cm}$ in the 1990 census from the analysis. After excluding these species, 370 species remained and represented 94\% (192,066 trees) of all woody trees with a diameter of $\geq 2 \mathrm{~cm}$. We generated a permuted community by shuffling the species identity of each tree. Data on actual and permuted communities have been deposited in the Dryad Digital Repository: http:// dx.doi.org/10.5061/dryad.bb460 (Kohyama et al. 2015). 


\section{Tree Size Distribution}

We quantified tree size distribution in stem diameter for the 1990 census using two coefficients. To represent the range of size distribution for trees of $\geq 2 \mathrm{~cm}$, we employed the upper 99th percentile stem diameter $(\mathrm{cm})$ to exclude the effect of tree density $N\left(\mathrm{ha}^{-1}\right)$, which differs among species (Kohyama et al. 2003). In accordance with Wright et al. (2003), we employed the third-moment coefficient of skewness with respect to logarithmic stem diameter to represent the shape of the size distribution. This skewness coefficient is also independent of population density $N$.

\section{Demographic Rates}

The time durations between the 1990 and 2000 censuses actually varied from 10 years and 9 days to 11 years and 90 days. We took this variation into account in the estimation of demographic parameters (Kubo et al. 2000). To estimate the tree size-dependent functions of mean growth rate $G(x)$ and mortality $M(x)$, we employed two hierarchical Bayesian models that simultaneously estimated the demographic parameters of all species based on the wholecommunity data. According to the plot census protocol (Condit 1998), the tag for a stem that died between censuses was reused for a living sprout from the same genet. From the point of view of stem-size dynamics with a sizecontinuous model given by equation (1), these situations are better dealt with as death-and-recruitment events rather than events of survival with abrupt size reduction. Therefore, we removed records of large size reductions (where the difference in logarithmic diameter between the two censuses was smaller than -0.1, i.e., an absolute decrease to $90.5 \%$ ) from demographic rate estimation. In total, 8,533 trees $(4.4 \%$ of all trees) fell into this category.

We estimated the mean growth rate, $G(x)\left(\mathrm{cm} \mathrm{year}^{-1}\right)$, as follows. We took into account two types of variation in observed size change between censuses: the measurement error and the within-population variation in latent growth rate (app. A). Following Kohyama et al. (2003), we approximated within-population variation in relative growth rate at size $x$ using an exponential distribution around the mean relative growth rate, $G(x) / x$. The tree size dependence of the mean relative growth rate has previously been fitted to either an exponential function (Kohyama et al. 2003) or a power function of $x$ (Muller-Landau et al. 2006). We employed a combination of these two as

$$
G(x) / x=a \exp (b x) x^{c},
$$

where $a\left(\right.$ year $\left.^{-1}\right), b\left(\mathrm{~cm}^{-1}\right)$, and $c\left([\operatorname{ln~} \mathrm{cm}]^{-1}\right)$ are speciesspecific parameters.

We estimated the mortality function, $M(x)\left(\right.$ year $\left.^{-1}\right)$, as follows. The latent death probability $p$ of a tree is the expectation of a binomial distribution of being either dead or alive during the census period. The instantaneous mortality $M(x)$ for a tree at $x(\mathrm{~cm})$ in the 1990 census, of which the intercensus interval is $\Delta t$ (year), satisfies the equation $p=1-\exp (-M(x) \Delta t)$. We employed the mortality function previously used by Rüger et al. (2011b) and Iida et al. (2014):

$$
M(x)=\alpha \exp (\beta x) x^{\gamma},
$$

where $\alpha\left(\right.$ year $\left.^{-1}\right), \beta\left(\mathrm{cm}^{-1}\right)$, and $\gamma\left([\operatorname{ln~} \mathrm{cm}]^{-1}\right)$ are speciesspecific parameters. Appendix A gives further details on the hierarchical Bayesian models used to estimate the parameters of $G(x)$ and $M(x)$.

We estimated the recruitment rate per unit area at the lowest diameter $x_{0}=2 \mathrm{~cm}, B$ (year ${ }^{-1} \mathrm{ha}^{-1}$ ), by employing the procedure of the Gf estimation (Kohyama and Takada 1998), where Gf implies "growth-by-frequency" from equation (2). Unlike the direct estimate based on the observation of recruitment events, the Gf estimate is free from unrecorded enter-and-die events that occur in a census interval. We obtained the recruitment rate for each species as the tree density at the size range of $1.5 \leq x<$ $2.5 \mathrm{~cm}\left(\mathrm{~cm}^{-1} \mathrm{ha}^{-1}\right)$ in the first census, multiplied by the mean growth rate of survivors $\left(\mathrm{cm} \mathrm{year}^{-1}\right)$ at $x_{0}=2 \mathrm{~cm}$ obtained using equation (5).

Because of the variation in census intervals from tree to tree, we could not estimate the intrinsic rate of increase in each species population, $r=(\mathrm{d} N / \mathrm{d} t) / N\left(\right.$ year $\left.^{-1}\right)$, by simply using the change in population density. Therefore, we determined the rate of population increase $r$ using $r=B / N-m$, where $B$ is the recruitment rate (year ${ }^{-1}$ $\left.\mathrm{ha}^{-1}\right), N$ is the population density at the first census $\left(\mathrm{ha}^{-1}\right)$, and $m$ is the population-average mortality (year ${ }^{-1}$ ) obtained from the observed tree size distribution and the estimated $M(x)$ of each species from equation (6).

\section{Linkage between Size Distribution and Demography}

We compared observed size distributions of actual and permuted communities with projected size distributions from demographic rates calculated using equation (4) under nonstationary and stationary assumptions. In the nonstationary population projection we used all of the estimated species-specific $G(x), M(x)$, and $r$, whereas in the stationary population projection we assumed that $r=0$ for all populations. Comparison of these two projections allows us to evaluate the contribution of population change to observed size distributions. For the convenience of simulating projected size distributions, we adopted equation (4) in terms of logarithmic size, $y=\ln x$, instead of $x$. The integration of equation (4) was carried out up to $x=250 \mathrm{~cm}$ because the observed maximum size of Neobalanocarpus heimii was $195.4 \mathrm{~cm}$ in the 1990 census. 
We present the dependence of each demographic rate on size-distribution coefficients (skewness and upper size) with linear model selection based on Akaike's information criterion, and we also provide conventional coefficients of determination $\left(R^{2}\right)$ for the cases where the linear (or loglinear) model was selected.

\section{Results}

The size distributions of species populations in the actual community varied widely in comparison with those in the permuted community with neutralized species properties (fig. B1 in app. B). In the actual community, skewness and upper size were varied across the entire range of population abundance, and the skewness coefficient was not correlated with upper size (fig. B1).

Growth and mortality parameters (eqq. [5], [6]) varied among species in the actual community (fig. $2 A-2 F$ ). For $54 \%$ of species (198), the parameters of the growth function were significantly separated in at least one model parameter from community-wide common parameters. Similarly, for $32 \%$ of species (119), the parameters of the mortality function were different from common parameters. Taken together, $23 \%$ of these species (86) had both growth and mortality parameters different and 62\% (231) had at least one growth or mortality parameter different from community common parameter(s). In contrast, the permuted community showed no evidence of interspecific variation (fig. $2 A-2 F$ ). The interspecific convergence of demographic parameters for the permuted community suggests that the community-wide estimation of mean growth rate and mortality with Bayesian models eliminates the effect of sampling bias associated with population density and tree size distribution.

We plotted the frequency distributions of per-density recruitment rate (fig. $2 G$ ), population average mortality (fig. $2 \mathrm{H}$ ), and intrinsic rate of population increase (fig. 2I). Interspecific variations in these rates in the actual community were substantially larger than those in the permuted community. For the actual community, there were three shortstatured species with extreme negative rates of population increase, $r<-0.05$ year $^{-1}$ (Pavetta graciliflora, Pavetta sp., and Macaranga hypoleuca). For the other 367 species, the rate of population increase ranged from -0.042 to 0.037 year $^{-1}$, with a median of almost $0\left(-6 \times 10^{-5}\right)$ year $^{-1}$ (fig. $2 I)$.

Estimated size-dependent curves of mean relative growth rate $G(x) / x$ (fig. $3 A$ ), mean absolute growth rate $G(x)$ (fig. $3 B$ ), and mortality $M(x)$ (fig. $3 C$ ) varied greatly from species to species in the actual community. Meanwhile, in the permuted community these curves were almost perfectly convergent among species (fig. $3 A-3 C$ ). Equation (3) shows that the projected size distribution is characterized by three demographic ratios, namely, the relative change with $x$ of growth rate $[\mathrm{d} G(x) / \mathrm{d} x] / G(x)$, the mortality-to-growth ratio $M(x) / G(x)$, and the population increase rate over growth rate $r / G(x)$. The larger these ratios are, the steeper the decline in tree size distribution with respect to size $x$, and thus the higher relative abundance of small-sized trees. Figure $3 D-3 F$ illustrates the size dependence of these ratios on the same scale. Curves of relative change of growth rate with $x$ were almost positive (fig. $3 D$ ), curves of mortality-to-growth ratio were always positive by definition (fig. $3 E$ ), and curves of population increase rate over growth rate were either negative or positive (fig. $3 F$ ) depending on the sign of $r$. Compared with the relative change of growth rate with $x$ (fig. $3 D$ ), the mortality-to-growth ratio and the population increase rate over growth rate (fig. $3 E, 3 F$ ) were more variable across species; thereby, the latter two ratios had the dominant effects on the projected size distributions. For all of these three $x$-dependent curves, the magnitude, as well as the degree of interspecific variation, was larger for smaller $x$ because of the property of increasing mean absolute growth rate with $x$ (fig. $3 B$ ).

Figure 4 demonstrates the comparison between observed size distributions and demographically projected size distributions in the actual and permuted communities. Among 370 species of the actual community, those for which the projected maximum stem diameter exceeded the simulation limit of $250 \mathrm{~cm}$ consisted of five species in the nonstationary population projection (Santiria rubiginosa, Scaphium macropodum, Shorea bracteolata, Shorea leprosula, and Shorea parvifolia) and eight species in the stationary population projection (Canarium megalanthum, Dipterocarpus cornutus, Dipterocarpus crinitus, Koompassia malaccensis, Millettia artopurpurea, Neobalanocarpus heimii, Parashorea densiflora, and Syzygium claviflora), all of which are emergent or canopy species. We excluded these in corresponding projections in figure 4 as well as in table 1. Projected size distributions of nonstationary and stationary populations reflected to a fair extent the observed size distributions of the actual community in terms of coefficient of skewness (fig. $4 A, 4 B$ ) and upper size (fig. $4 D, 4 E$ ), as the foliage partitioning theory predicts. The projected nonstationary size distributions fitted the observed distributions better (with about twice the $R^{2}$ values) than the projected stationary distributions in the actual community. In the case of the permuted community, all projections generated convergent size distributions among species regardless of a wider variation in observed distributions (fig. 4, gray symbols). This was due to the convergent growth and mortality curves among species (fig. 3 , gray lines).

The intrinsic rate of population increase alone did not explain the observed interspecific variation in size distri- 

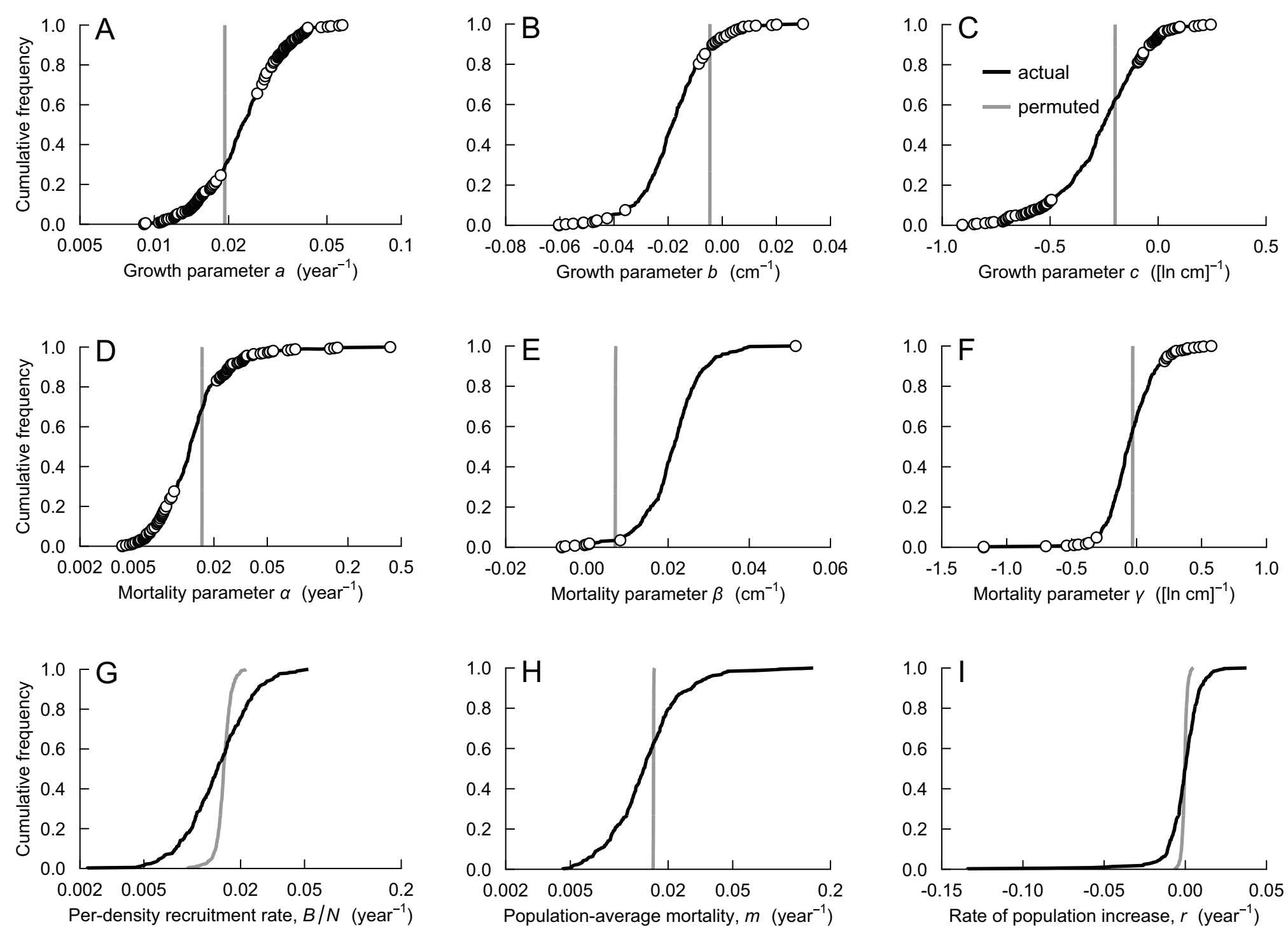

Figure 2: Cumulative frequency distributions of 370 species for estimated demographic parameters and rates. $A-C$, Parameters of the growth rate function (eq. [5]). $D-F$, Parameters of the mortality function (eq. [6]). G, Per-density recruitment rate $(B / N) . H$, Population-average mortality $(m) . I$, Intrinsic rate of population increase $(r=B / N-m)$. Black lines show the actual community, and gray lines show the permuted community. Open circles in $A-F$ indicate species for which parameters are significantly different from the community-wide common parameter $(P<.05)$. Distributions in positive parameters and rates (i.e., $A, D, G$, and $H$ ) are shown on a logarithmic scale. 

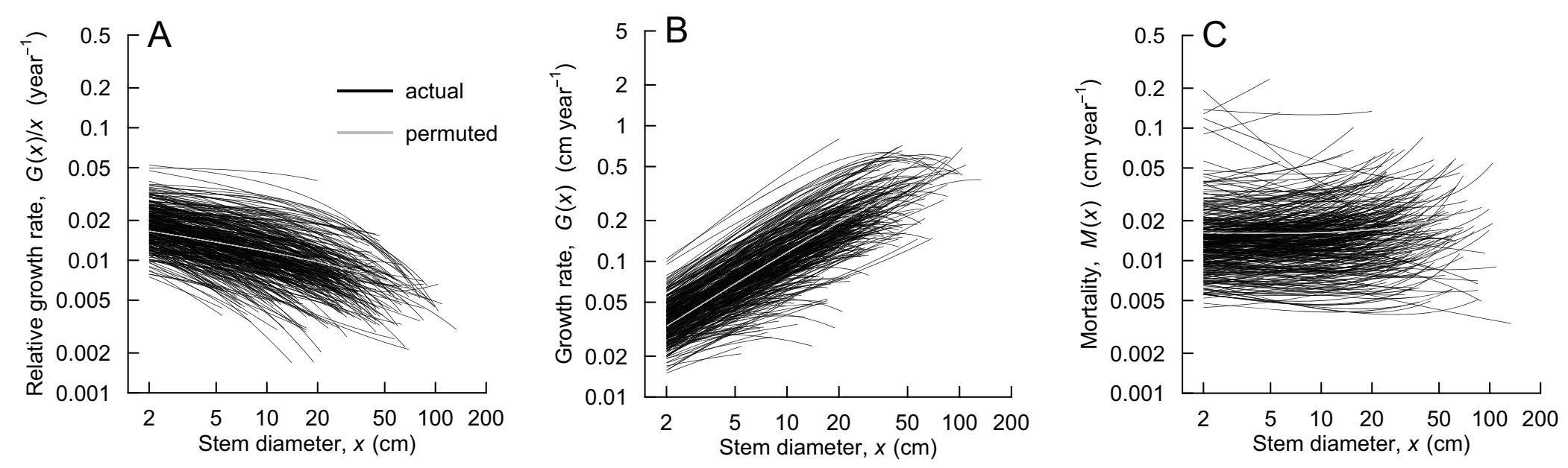

$\stackrel{w}{\omega}$
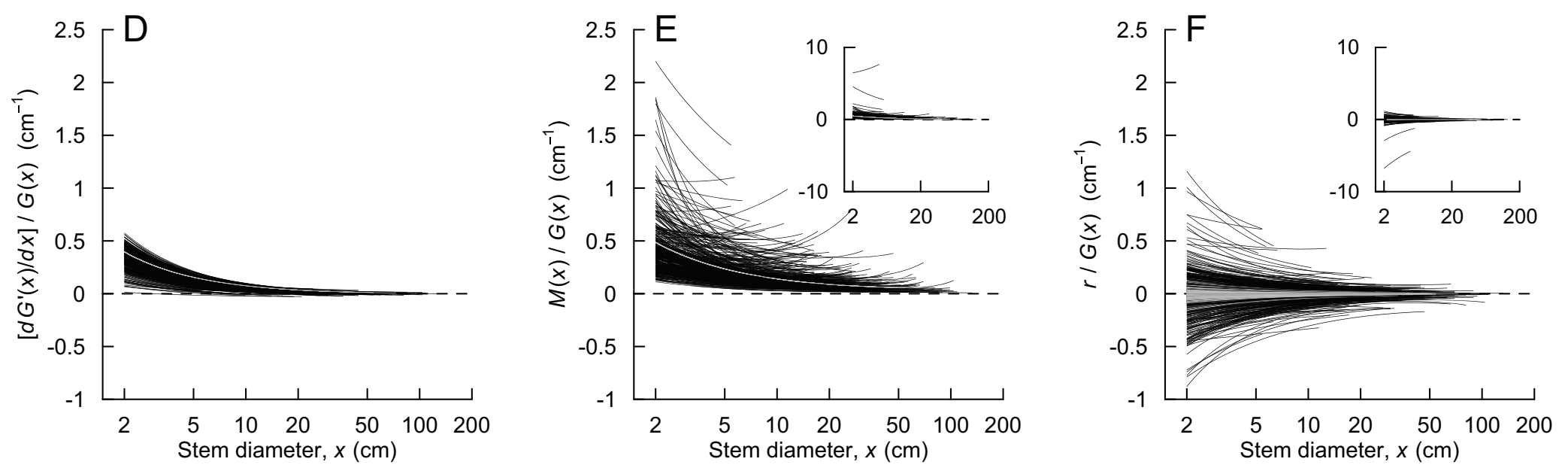

Figure 3: Dependence of size growth rate and mortality on tree size $x$ in stem diameter for 370 species in the Pasoh forest. $A$, Mean relative growth rate $G(x) / x$. $B$, Mean absolute growth rate $G(x)$. C, Mortality $M(x)$. D, Relative change in growth rate with $x[\mathrm{~d} G(x) / \mathrm{d} x] / G(x)$. E, Mortality-to-growth ratio $M(x) / G(x)$. $F$, Population increase rate over growth rate $r / G(x)$. Black lines indicate the actual community and gray lines indicate the permuted community throughout the range to the upper size of each species. Vertical axes of $D-F$ are on the same scale; insets in $E$ and $F$ include two outlier species, Pavetta graciliflora and Pavetta sp. 

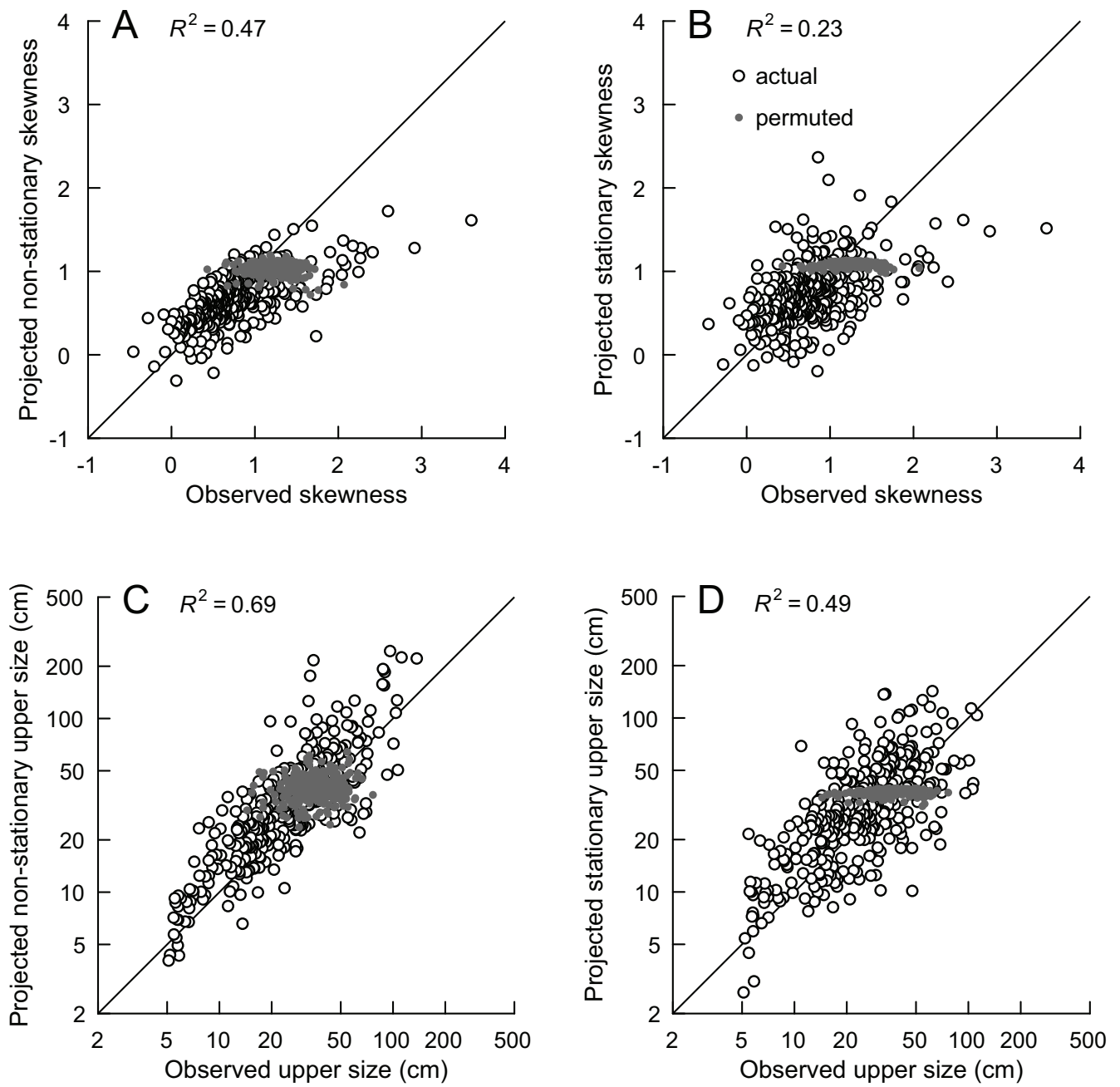

Figure 4: Comparison of observed and projected stem diameter distributions of the Pasoh tree community, quantified by the coefficient of skewness in terms of logarithmic stem diameter $(A, B)$ and the upper size defined by 99th percentile stem diameter $(C, D)$. Open circles show species in the actual community, and gray circles show those in the permuted community. $A$, $C$, Projected nonstationary populations (with observed rate of population increase $r$ ). B, D, Projected stationary populations (assuming $r=0$ ). Diagonal lines show $1: 1$ lines of perfect agreement, whereas $R^{2}$ values are for $(\log )$ linear models for the case of the actual community. Five species in the nonstationary projection and eight species in the stationary projection from the actual community were excluded because their projected maximum diameters were larger than the upper boundary diameter of $250 \mathrm{~cm}$ in the simulations with equation (4).

bution (table 1). In contrast, a large per-density recruitment rate was correlated with high skewness and low upper size (table 1), as the foliage partitioning theory predicts. Interspecific variation in mortality-to-growth ratio at small $x$ (fig. $3 E$ ) contributed to the variation in projected size distributions. As expected from equation (3), large mortalityto-growth ratio (indicated logarithmically by $\ln M(x)-$ $\ln G(x)$ in table 1) at small $x$ was associated with high skewness and low upper size (table 1). Table 1 further shows that high mortality-to-growth ratio was realized by the combination of high mortality and low growth rate.

There was an interspecific positive correlation between logarithmic mean growth rate and logarithmic mortality for the range $2 \leq x \leq 9 \mathrm{~cm}(P<.05)$, indicating a growth-survival trade-off. This correlation was highest at $x=2 \mathrm{~cm}\left(P=10^{-5}\right)$, for which $R^{2}$ was only 0.05 (fig. $5 A$ ). In contrast, the major demographic determinant of size distribution variation was along the independent (or orthogonal) axis of mortality-to-growth ratio for small tree sizes (fig. 3E). Low skewness was associated with low mortality-to-growth ratio at $x=2 \mathrm{~cm}$ in the observed populations (fig. $5 B$ ) and more strongly in projected stationary populations (fig. $5 C$ ), as the foliage partitioning theory predicts (fig. $1 A$ ). In addition, the rate of population increase tended to be higher for species with low juvenile mortality (fig. 5A). When the mortality-to-growth ratios 
Table 1: Correlations between demographic rates and size distribution

\begin{tabular}{|c|c|c|c|c|}
\hline \multirow[b]{2}{*}{ Demographic rate } & \multicolumn{4}{|c|}{ Dependence on size-distribution skewness $S$ and upper size $U$} \\
\hline & Observed populations & $R^{2}$ & Stationary populations & $R^{2}$ \\
\hline$r$ & -.0011 & $\cdots$ & $.0015-.016 S+.0028 \ln U$ & .29 \\
\hline $\ln B-\ln N$ & $-3.8+.40 S-.23 \ln U$ & .26 & $-4.3+.88 S-.18 \ln U$ & .58 \\
\hline $\ln G(2)$ & $-3.6-.24 S+.15 \ln U$ & .20 & $-3.6-.20 S+.15 \ln U$ & .18 \\
\hline $\ln M(2)$ & $-4.5+.27 S$ & .06 & $-5.1+1.1 S$ & .57 \\
\hline $\ln G(5)$ & $-3.5-.16 S+.29 \ln U$ & .26 & $-3.9+.12 S+.35 \ln U$ & .29 \\
\hline $\ln M(5)$ & $-3.9+.25 S-.18 \ln U$ & .09 & $-4.0+.88 S-.26 \ln U$ & .62 \\
\hline $\ln G(10)$ & $-3.5-.075 S+.41 \ln U$ & .31 & $-4.3+.41 S+.51 \ln U$ & .40 \\
\hline $\ln M(10)$ & $-3.4+.23 S-.31 \ln U$ & .15 & $-3.2+.70 S-.45 \ln U$ & .65 \\
\hline
\end{tabular}

Note: Results of the model selection for the dependence of each demographic rate on the coefficient of skewness, $S$, and the upper size, $U(\mathrm{~cm})$, of stem diameter distribution are shown for observed and projected stationary populations in the actual community. Demographic rates are the rate of population increase, $r\left(\right.$ year $\left.^{-1}\right)$; recruitment rate $(B)$ per population density $(N), B / N\left(\right.$ year $\left.^{-1}\right)$; mean size growth rate, $G(x)\left(\mathrm{cm} \mathrm{year}^{-1}\right)$; and instantaneous rate of mortality, $M(x)$ (year ${ }^{-1}$ ), at $x=2,5$, and $10 \mathrm{~cm}$. All positive variables (except $S$ and $r$ ) were log transformed. For stationary populations, those eight species for which the projected maximum diameter was larger than the simulation upper boundary of $250 \mathrm{~cm}$ (shown in the main text) were excluded.

of juveniles were similar, size-distribution skewness tended to be higher for species with a higher rate of population increase (fig. $5 B$ ). The observed skewness was better explained by the linear combination of logarithmic mortality-to-growth ratio at $2 \mathrm{~cm}$ and the rate of population increase $\left(R^{2}=0.35\right)$ than by the linear function of log mortality-to-growth ratio alone $\left(R^{2}=0.19\right)$. These findings partly support the prediction of successional niche theory (fig. 1B).

\section{Discussion}

In this study, we examined the degree to which vertical heterogeneity in stand structure enhances tree species coexistence in a tropical forest tree community. Our results showed that interspecific variation in tree size distribution reflected the variation in demographic properties, as predicted by the foliage partitioning theory (fig. $1 \mathrm{~A}$ ), but not, apparently, the growth-survival trade-off (fig. 1B). Low mortality together with high mean growth rate, particularly for small tree sizes, contributed to low size-distribution skewness (table 1). Observed size distributions, characterized by skewness coefficient and upper size, were correlated with projected size distributions (fig. 4).

Previously, Muller-Landau et al. (2006) applied equation (4) to all trees, ignoring species differences, and found that projected stationary size distribution from size-dependent growth rate and mortality satisfactorily reproduced the observed size distribution in Pasoh and BCI forests. This suggests that the community-wide tree size distributions were in demographic equilibrium. In contrast, in this study, looking at individual tree species, we found large interspecific variations in observed size distributions and demographic rates (figs. 2-4), such that they were largely divergent from demographic neutrality as represented by the permuted community. The rate of population increase $r$ was also largely varied among species (fig. $2 I$ ). Therefore, the Pasoh forest exhibited a temporal change in species abundance, keeping the community-wide tree size distribution unchanged with time. The rate of population increase $r$ alone showed no relationship with either size distribution skewness or upper size (table 1). However, the projected nonstationary size distributions with the observed $r$ fit the observed distributions better than the projected stationary distributions with $r=0$ (fig. 4). Interspecific variation in the mortality-to-growth ratio mainly explained the variation in observed skewness, as expected by foliage partitioning theory (table 1; fig. $5 B$ ); meanwhile, interspecific variation in $r$ additionally contributed to the observed skewness variation (fig. $5 B$ ).

Although equation (4) projects a unique size distribution by using a set of demographic rates, the projected distribution from equation (4) does not indicate the absolute quantity of these rates: the projected size distribution is unchanged whenever all of these rates are multiplied by any positive factor. In spite of this theoretical relationship between demographic rates and projected size distributions, we can expect demographic similarity and trade-offs in life-history performance among coexisting tree species because of underlying physiological and environmental constraints. We indeed found that low sizedistribution skewness was realized by the combination of low mortality and high growth rate, and such an advantage in growth and survival for species with low skewness was balanced with a low per capita recruitment rate (table 1). Populations with low size-distribution skewness had a higher relative abundance of large-sized adult trees than populations with high skewness and a low abundance of 

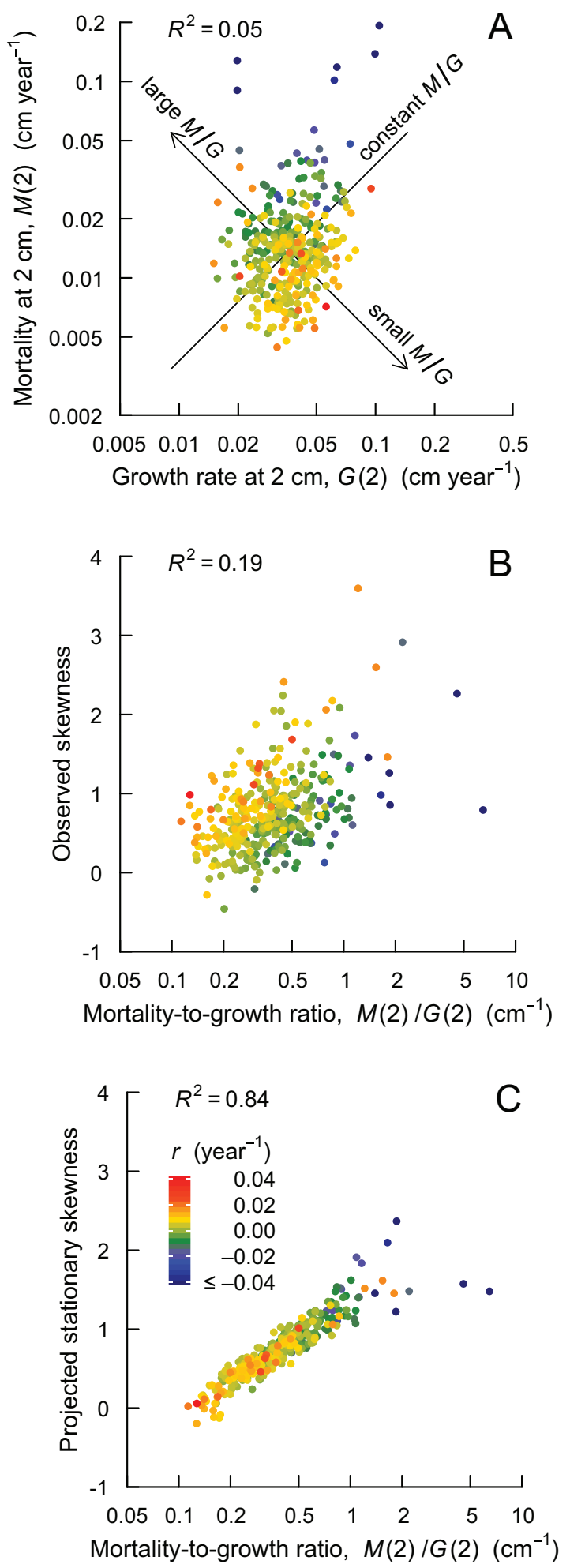

Figure 5: $A$, Relationship between growth rate $G(x)$ and mortality $M(x) . B$, Dependence of the coefficient of skewness of observed populations on the mortality-to-growth ratio. $C$, Dependence of the coefficient of skewness of stationary populations on the mortality-to- adult trees. Therefore, compared with the recruitment rate per population density $(B / N)$, the fecundity per adult tree density was still lower for species with a high growth rate and a high probability of survival.

Besides the variation in the skewness of size distribution, the observed large variation in upper size was also explained by demographic projections (fig. $4 C, 4 D$ ). Tallstatured species showed a high growth rate, low mortality, and low fecundity, whereas low-statured species showed the opposite demographic properties (table 1). This demographic trade-off between tree vitality (in growth and survival) and fecundity supported the prediction of the foliage partitioning theory for stable species coexistence by differentiating maximum adult size (Kohyama and Takada 2009), which has also been supported empirically (Thomas 1996; Kohyama et al. 2003; King et al. 2006a, 2006b; Poorter et al. 2008; Iida et al. 2014). We found that differentiations in size-distribution skewness and upper size were associated with similar demographic properties, while we also detected that the low skewness was principally explained by low mortality-to-growth ratio in small tree sizes, whereas large upper size was mainly related to high growth rate, particularly of large-sized trees (table 1). Because there were large interspecific variations both in upper size and skewness of size distribution and because there was no correlation between upper size and skewness, the two mechanisms of the foliage partitioning theory appear to be working together to enhance stable species coexistence in the tree community of the Pasoh forest.

Our findings stand in contrast to those of Wright et al. (2003), who examined the growth-survival trade-off in the BCI forest. They reported that high growth rate and highnot low-mortality in juveniles with $1-5-\mathrm{cm}$ stem diameters were associated with low size-distribution skewness, supporting successional niche theory in a temporally changing community (fig. $1 B$ ). It is possible that the effect of the selective decline of light-demanding species and the increase of shade-tolerant species in the BCI forest is larger than the effect of demographic differentiation among stable populations. Indeed, Condit et al. (1999) suggested that the tree community of the Pasoh forest appears to be more stably maintained with a denser canopy and a lower frequency of light-demanding species than the community at BCI. However, there was no correlation between the shape of the size distribution and the population increase rate (Condit et al. 1998). Condit et al. (1998) analyzed species' size distributions in the same 50-ha plot in BCI using a size-class transition model at $5-\mathrm{cm}$-diameter classes. They found that the

growth ratio, represented at stem diameter of $x=2 \mathrm{~cm}$ for the actual community. The color of symbols indicates the rate of population increase $r$, from dark blue for small $r$ to orange red for large $r$. 
shape of the size distribution was related to the transition rate at the smallest size class threshold of $5 \mathrm{~cm}$ but not to (population-average) mortality nor to the rate of population increase, which are partly in accordance with the present results. These previous studies estimated demographic rates separately for each of the abundant species; therefore, further intensive community-wide analysis is needed to compare the Pasoh and BCI forests.

In horizontally heterogeneous gap-dynamic forests, demographic variations among species (Pacala and Rees 1998) and those within species (Clark et al. 2010) have been shown to facilitate species coexistence. Actually, Iida et al. (2014) and this study (fig. 5A) found a weak indication of a growth-survival trade-off for small-sized trees in the Pasoh forest. However, the remaining variation independent of growth-survival trade-off-that is, interspecific variation in mortality-to-growth ratio-mainly explained the observed variation in size distribution (table 1; fig. $5 B$ ), which supports species coexistence via foliage partitioning mechanisms. The apparent weak or no correlation between growth rate and mortality therefore implies the existence of multiple overlaid trade-offs corresponding to the two alternative theories of foliage partitioning and successional niche (fig. 1). We conclude that mechanisms relying on the partitioning of vertical light gradient among species work together with the mechanisms relying on the partitioning of horizontally heterogeneous light conditions to promote species coexistence in species-rich tropical forests.

\section{Acknowledgments}

We acknowledge that the Pasoh 50-ha Forest Dynamics Plot is a collaborative project of the Forest Research Institute Malaysia; the National Institute for Environmental Studies, Japan; and the Center for Tropical Forest Science, Smithsonian Tropical Research Institute. We thank T. Kubo and Y. Iida for fruitful suggestions and M. Eichhorn and another reviewer for essential comments. A Bullard Fellowship from Harvard University to T.S.K. in 19981999 helped us start this study, and a Grant-in-Aid for Scientific Research from the Japan Society for the Promotion of Science (24247003) helped us finalize it.

\section{APPENDIX A \\ Estimation of Parameters for Growth and Mortality Functions}

We carried out Markov chain Monte Carlo (MCMC) simulations for the hierarchical Bayesian models to estimate parameters of growth rate function (eq. [5]) and mortality function (eq. [6]). We explain only the case of growth function here, because the case of mortality function is similar.

The observed change in each tree in terms of logarithmic stem diameter, $\ln x$, was assumed to have a normally distributed measurement error around its latent value with a standard error of $10^{-2}$. Within-population variation in latent relative growth rate at $x$ was approximated using an exponential distribution. As the latent mean growth rate of a tree at size $x, G(x)$, is defined to be positive, we took the logarithm for both sides of equation (5) to estimate growth parameters:

$$
\ln G(x)-\ln x=A+b x+c \ln x
$$

where $A=\ln a$. Each of the parameters $A, b$, and $c$ of equation (A1), which are specific to each species, was set to be the sum of the "common" parameter for all 370 species and the "species-difference" parameter for each species. We set the prior distributions of common parameters to be a noninformative normal distribution with a mean of 0 and a standard deviation of $10^{2}$. Each of the prior distributions of species-difference parameters was set to be a normal distribution with a mean of 0 and a standard deviation of $\sigma$ as the hyperparameter, and the prior distribution of the hyperparameter $\sigma$ was set to be a noninformative uniform distribution between 0 and $10^{2}$.

We obtained the posterior distributions for the parameters of the growth and mortality models using MCMC simulations. We used JAGS (ver. 3.3.0; Plummer 2011) via the R2jags package ( $\mathrm{Su}$ and Yajima 2012) in $\mathrm{R}$ (ver. 3.0.1; R Development Core Team 2013). Codes for the growth and mortality models, together with the data sets analyzed, are available in the Dryad Digital Repository: http://dx.doi.org/10.5061/dryad.bb460 (Kohyama et al. 2015). In each model with the actual community data, we ran three independent Markov chains of 50,000 steps with sampling every 40 steps. We discarded the first 10,000 steps as burn-in. For the cases of the permuted community, we used about three times larger steps, because almost no interspecific difference existed. The convergence and stationarity of the Markov chains were checked using Gelman and Rubin's diagnostics (Gelman et al. 2003). As estimates of model parameters $A$ (and thus $a), b$, and $c$, we employed the sum of the medians of the simulated posterior distributions of their common and species-difference parameters, whereas we deemed the deviation of the model parameters of each species from the community-wide common parameters to be significant when the $95 \%$ credible interval of the posterior distribution of species-difference parameters did not include 0 . 


\section{APPENDIX B}

\section{Interspecific Variation in Size-Distribution Measures}
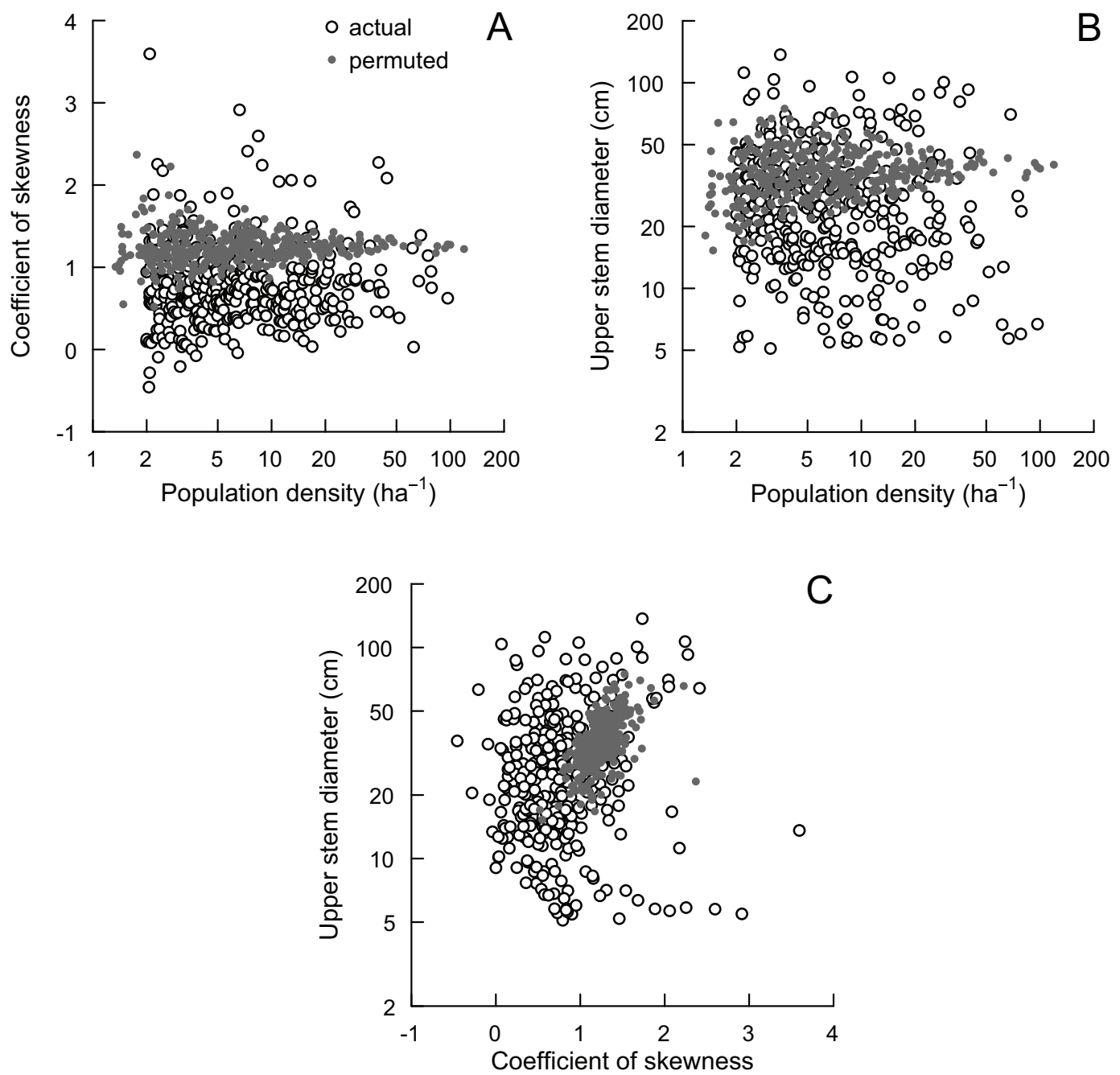

Figure B1: Population density and size-distribution coefficients of 370 tree species in the Pasoh forest. A, Population density versus skewness coefficient in terms of logarithmic stem diameter. $B$, Population density versus 99th percentile upper size in stem diameter. $C$, Skewness coefficient versus upper size. Open circles show species in the actual community, and gray circles show species in the permuted community.

\section{Literature Cited}

Bin, Y., W. Ye, H. C. Muller-Landau, L. Wu, J. Lian., and H. Cao. 2012. Unimodal tree size distributions possibly result from relatively strong conservatism in intermediate size classes. PLoS ONE 7:e52596.

Clark, J. S., D. Bell, C. Chu, B. Courbaud, M. Dietze, M. Hersh, J. HilleRisLambers, et al. 2010. High-dimensional coexistence based on individual variation: a synthesis of evidence. Ecological Monographs 80:569-608.

Condit, R. 1998. Tropical forest census plots. Springer, Berlin.

Condit, R., P. S. Ashton, N. Manokaran, J. V. LaFrankie, S. P. Hubbell, and R. B. Foster. 1999. Dynamics of the forest communities at Pasoh and Barro Colorado: comparing two 50-ha plots. Philosophical Transactions of the Royal Society B: Biological Sciences 354:1739-1748.

Condit, R., R. Sukumar, S. P. Hubbell, and R. B. Foster. 1998. Predicting population trends from size distributions: a direct test in a tropical tree community. American Naturalist 152:495-509.

Falster, D. S., and M. Westoby. 2005. Alternative height strategies among 45 dicot rain forest species from tropical Queensland, Australia. Journal of Ecology 93:521-535.

Feeley, K. J., S. J. Davies, M. N. Nur Supardi, A. R. Kassim, and S. 
Tan. 2007. Do current stem size distributions predict future population changes? an empirical test of intraspecific patterns in tropical trees at two spatial scales. Journal of Tropical Ecology 23:191198.

Gelman, A., J. B. Carlin, H. S. Stern, and D. B. Rubin. 2003. Bayesian data analysis. 2nd ed. Chapman \& Hall/CRC, Boca Raton, FL.

Hara, T. 1984. A stochastic model and the moment dynamics of the growth and size distribution in plant populations. Journal of Theoretical Biology 109:173-190.

Iida, Y., T. S. Kohyama, T. Kubo, A. R. Kassim, L. Poorter, F. J. Sterck, and M. D. Potts. 2011. Tree architecture and life-history strategies across 200 co-occurring tropical tree species. Functional Ecology 25:1260-1268.

Iida, Y., L. Poorter, F. J. Sterck, A. R. Kassim, M. D. Potts, T. Kubo, and T. S. Kohyama. 2014. Linking size-dependent growth and mortality with architectural traits across 145 co-occurring tropical tree species. Ecology 95:353-363.

King, D. A., S. J. Davies, and M. N. Nur Supardi. 2006a. Growth and mortality are related to adult tree size in a Malaysian mixed dipterocarp forest. Forest Ecology and Management 223:152-158.

King, D. A., S. J. Wright, and J. H. Connell. 2006b. The contribution of interspecific variation in maximum tree height to tropical and temperate diversity. Journal of Tropical Ecology 22:11-24.

Kochummen, K. M. 1997. Tree flora of Pasoh forest. Forest Research Institute Malaysia, Kepon.

Kohyama, T. 1992. Size-structured multi-species model of rain forest trees. Functional Ecology 6:206-212.

1993. Size-structured tree populations in gap-dynamic forest-the forest architecture hypothesis for the stable coexistence of species. Journal of Ecology 81:131-143.

Kohyama, T., and T. Hara. 1989. Frequency distribution of tree growth rate in natural forest stands. Annals of Botany 64:47-57.

Kohyama, T., T. Kubo, and E. Macklin. 2005. Effect of temporal autocorrelation on apparent growth rate variation in forest tree census data and an alternative distribution function of tree growth rate. Ecological Research 20:11-15.

Kohyama, T., E. Suzuki, T. Partomihardjo, T. Yamada, and T. Kubo. 2003. Tree species differentiation in growth, recruitment and allometry in relation to maximum height in a Bornean mixed dipterocarp forest. Journal of Ecology 91:797-806.

Kohyama, T., and T. Takada. 1998. Recruitment rates in forest plots: Gf estimates using growth rates and size distributions. Journal of Ecology 86:633-639.

- 2009. The stratification theory for plant coexistence promoted by one-sided competition. Journal of Ecology 97:463-471.

- 2012. One-sided competition for light promotes coexistence of forest trees that share the same adult height. Journal of Ecology 100:1501-1511.

Kohyama, T. S., M. D. Potts, T. I. Kohyama, Abd Rahman K., and P. S. Ashton. 2015. Data from: Demographic properties shape tree size distribution in a Malaysian rain forest. American Naturalist, Dryad Digital Repository, http://dx.doi.org/10.5061/dryad.bb460.

Kubo, T., T. Kohyama, M. D. Potts, and P. S. Ashton. 2000. Mortality rate estimation when inter-census intervals vary. Journal of Tropical Ecology 16:753-756.
Muller-Landau, H. C., R. S. Condit, K. E. Harms, C. O. Marks, S. C. Thomas, S. Bunyavejchewin, G. Chuyong, et al. 2006. Comparing tropical forest tree size distributions with the predictions of metabolic ecology and equilibrium models. Ecology Letters 9: 589-602.

Pacala, S. W., and M. Rees. 1998. Models suggesting field experiments to test two hypotheses explaining successional diversity. American Naturalist 152:729-737.

Plummer, M. 2011. Just Another Gibbs Sampler. Version 3.3.0. http://mcmc-jags.sourceforge.net/. Accessed July 21, 2013.

Poorter, L., S. J. Wright, H. Paz, D. D. Ackerly, R. Condit, G. IbarraManríquez, K. E. Harms, et al. 2008. Are functional traits good predictors of demographic rates? evidence from five Neotropical forests. Ecology 89:1908-1920.

R Development Core Team. 2013. R: a language and environment for statistical computing. R Foundation for Statistical Computing, Vienna. http://www.R-project.org/. Accessed July 21, 2013.

Rees, M., R. Condit, M. Crawley, S. Pacala, and D. Tilman. 2001. Long-term studies of vegetation dynamics. Science 293:650-655.

Rüger, N., U. Berger, S. P. Hubbell, G. Vieilledent, and R. Condit. 2011a. Growth strategies of tropical tree species: disentangling light and size effects. PLoS ONE 6:e25330.

Rüger, N., A. Huth, S. P. Hubbell, and R. Condit. 2011b. Determinants of mortality across a tropical lowland rainforest community. Oikos 120:1047-1056.

Shea, K. L. 1985. Demographic aspects of coexistence in Engelmann spruce and subalpine fir. American Journal of Botany 72:18231833.

Su, Y.-S., and M. Yajima. 2012. R2jags: a package for running jags from R. R package, version 0.03-08. http://CRAN.R-project.org/ package $=$ R2jags. Accessed July 21, 2013.

Suzuki, T. 1966. Forest transition as a stochastic process I [in Japanese]. Journal of the Japanese Forestry Society 48:436-439.

Takahashi, K. 1996. Plastic response of crown architecture to crowding in understorey trees of two co-dominating conifers. Annals of Botany 77:159-164.

Takahashi, K., and T. Kohyama. 1999. Size-structure dynamics of two conifers in relation to understorey dwarf bamboo: a simulation study. Journal of Vegetation Science 10:833-842.

Thomas, S. C. 1996. Asymptotic height as a predictor of growth and allometric characteristics in Malaysian rain forest trees. American Journal of Botany 83:556-566.

VanSickle, J. 1977. Analysis of a distributed-parameter population model based on physiological age. Journal of Theoretical Biology 64:571-586.

Wright, S. J., K. Kitajima, N. J. B. Kraft, P. B. Reich, I. J. Wright, D. E. Bunker, R. Condit, et al. 2010. Functional traits and the growth-mortality trade-off in tropical trees. Ecology 91:3664-3674.

Wright, S. J., H. C. Mueller-Landau, R. Condit, and S. P. Hubbell. 2003. Gap-dependent recruitment, realized vital rates, and size distributions of tropical trees. Ecology 84:3174-3185.

Associate Editor: Jürgen Groeneveld Editor: Susan Kalisz 